\title{
How Useful Are Blood and Stool Biomarkers for Detecting Subclinical Mucosal Inflammation in Ulcerative Colitis?
}

\author{
Byong Duk Ye
}

Received: 5 December 2013/Accepted: 5 December 2013/Published online: 17 January 2014

(c) Springer Science+Business Media New York 2014

Endoscopy is essential for correct diagnosis and evaluation of the disease activity of ulcerative colitis (UC). Although the clinical symptoms of UC, for example mucoid diarrhea, bleeding, and abdominal pain can improve after appropriate therapy for active UC, inflammatory lesions can still be observed even in clinical remission. Stated differently, in a substantial number of cases, clinical symptoms and endoscopic lesions do not correlate. Furthermore, as the concept of endoscopic mucosal healing $(\mathrm{MH})$ as a therapeutic target of inflammatory bowel disease gains acceptance, objective evaluation of inflammation is being increasingly emphasized. In contrast with Crohn's disease (CD), which can involve the entire gastrointestinal tract, mucosal lesions of UC can more easily be evaluated endoscopically, because the inflammation is limited to the colon or rectum. Therefore, $\mathrm{MH}$ seems to be a more realistic therapeutic target in UC than in CD. Nevertheless, considering the discomfort of patients, the high cost of examination, and the potential complications of invasive procedures, well-validated surrogate markers for endoscopic lesions are needed. Although C-reactive protein (CRP) and erythrocyte sedimentation rate (ESR) are nonspecific biomarkers of inflammation, they can be readily measured, serving as potential markers for mucosal inflammation in UC.

Since mucosal hyperemia and granularity were used for endoscopic classification of UC patients in a pivotal study by Truelove et al. [1], several endoscopic scoring systems

\footnotetext{
B. D. Ye $(\bowtie)$

Department of Gastroenterology and Inflammatory Bowel Disease Center, Asan Medical Center, University of Ulsan College of Medicine, 88, Olympic-ro 43-gil, Songpa-gu, Seoul 138-736, Republic of Korea

e-mail: bdye@amc.seoul.kr
}

have been adopted in clinical research and practice. A decade after Truelove's study was published, the Baron Score was developed in 1964. Soon after, the Powell-Tuck Score, the Mayo Endoscopic Score, the Rachmilewitz Endoscopic Index, Hanauer's Sigmoidoscopic Index, and the modified Baron Score were introduced. Several studies have investigated the correlation of blood biomarkers and these endoscopic indices. In 2008, Osada et al. [2] scored the endoscopic activity in seven colonic segments (appendiceal region, cecum, ascending colon, transverse colon, descending colon, sigmoid colon, and rectum) by use of the Mayo Endoscopic Score, comparing endoscopic activity with blood biomarkers. They reported that CRP and ESR correlated well with proximal rather than distal colonic activity, suggesting that total colonoscopy is needed in cases of elevated CRP or ESR among UC patients in clinical remission [2]. Karoui et al. [3] also reported a significant correlation between CRP and the Rachmilewitz Endoscopic Index $(r=0.46, P<0.0001)$ in a prospective study of 101 UC patients. In a population-based study (the Inflammatory Bowel South-Eastern Norway II) of 61 newly diagnosed UC patients, the difference in CRP was significant between a Mayo Endoscopic Score of 1 and a Score of $3(P=0.007)$, and between Score 1 and a combination of Scores 2 and $3(P=0.016)$ [4]. Nevertheless, no prior studies have evaluated the correlation of blood biomarkers and the various endoscopic scoring systems at the same time.

In this issue of Digestive Diseases and Sciences, Yoon et al. [5] investigated the correlation between blood inflammatory markers and endoscopic severity indices among patients with UC. They evaluated over 700 endoscopies, using five widelyused endoscopic scoring systems: the Powell-Tuck Score, the Mayo Endoscopic Score, the Rachmilewitz Endoscopic Index, Hanauer's Sigmoidoscopic Index, and the modified 
Baron Score. As a result, correlation coefficients between endoscopic activity indices and CRP, ranging from $r=0.457$ to $r=0.523$, and ESR ranging from $r=0.342$ to $r=0.435$, were observed. Moreover, CRP, with a cutoff of $\leq 8 \mathrm{mg} / \mathrm{L}$, had a sensitivity ranging from 50.5 to $53.3 \%$ and a specificity ranging from 85.1 to $87.2 \%$ for detection of endoscopic remission using the five endoscopic indices. ESR, with a cutoff of $\leq 20 \mathrm{~mm} / \mathrm{h}$, had a sensitivity range of $68.7-71.3 \%$ and a specificity range of 63.4-66.4\%. Therefore, they concluded that despite the modest correlation of CRP and ESR with endoscopic activity indices among UC patients, the low sensitivities of CRP and ESR for detecting endoscopic remission suggest that neither CRP nor ESR alone is sufficient. Despite of the study's limitations, including its retrospective design, single center-based research, and vague presentation of methodology for analyzing endoscopic images, this study suggests there are important the limitations to CRP and ESR as biomarkers reflecting endoscopic features in UC. More sensitive and specific biomarkers for endoscopic lesions are needed for UC patients.

Recently, the performance characteristics of fecal protein biomarkers, for example lactoferrin, calprotectin, and polymorphonuclear neutrophil elastase, have been investigated. A significant correlation was identified between endoscopic inflammation measured by the Mayo Endoscopic Score and fecal lactoferrin $(r=0.56, P<0.001)$, fecal calprotectin $(r=0.49, P<0.001)$, and polymorphonuclear neutrophil elastase $(r=0.36, \quad P<0.05)$, whereas CRP $(r=0.23, P>0.05)$ was not significantly correlated [6]. The Rachmilewitz Endoscopic Index also correlated better with fecal calprotectin $(r=0.834)$ than the Rachmilewitz Clinical Activity Index $(r=0.672)$ and CRP $(r=0.503)$ [7]. The overall accuracy of detection of endoscopically active disease (Rachmilewitz Endoscopic Activity Score 4) was reported to be $89 \%$ for calprotectin, $73 \%$ for the Clinical Activity Index, and $62 \%$ for elevated CRP [7]. Fecal calprotectin, also, had a better correlation with the modified Baron Score $(r=0.821)$ than with the Lichtiger Index $(r=0.682)$ and CRP $(r=0.556)$ [8]. These results suggest that among UC patients, fecal biomarkers better reflect endoscopic inflammation than do blood biomarkers.

The five endoscopic indices studied in Yoon's paper were not developed by use of fundamental statistical methods of index development, nor have they been properly validated. Recently, the ulcerative colitis endoscopic index of severity (UCEIS) and the ulcerative colitis colonoscopic index of severity (UCCIS), new indices for endoscopic grading of ulcerative colitis, were developed using current principles of index development and digital technology. These indices have also been validated in subsequent studies $[9,10]$, although additional studies are needed to test the validity and usefulness of UCEIS and UCCIS in heterogeneous patient groups. Furthermore, considering the patchy distribution of inflammatory lesions in some patients with UC, total colonic evaluation using UCCIS may reflect more accurate assessment of the inflammatory burden of UC.

In summary, Yoon et al. [5] report the usefulness and the limitations of CRP and ESR as surrogate markers of colonic and rectal mucosal inflammation in UC patients. More useful and easy-to-use markers reflecting endoscopic activity of UC are still needed for the future management of UC patients.

Conflict of interest None.

\section{References}

1. Truelove SC, Witts LJ. Cortisone in ulcerative colitis. Final report on a therapeutic trial. Br Med J. 1955;2:1041-1048.

2. Osada T, Ohkusa T, Okayasu I, et al. Correlations among total colonoscopic findings, clinical symptoms, and laboratory markers in ulcerative colitis. J Gastroenterol Hepatol. 2008;23: S262-S267.

3. Karoui S, Laz S, Serghini M, Bibani N, Boubaker J, Filali A. Correlation of C-reactive protein with clinical and endoscopic activity in patients with ulcerative colitis. Dig Dis Sci. 2011;56: 1801-1805.

4. Ricanek P, Brackmann S, Perminow G, et al. Evaluation of disease activity in IBD at the time of diagnosis by the use of clinical, biochemical, and fecal markers. Scand J Gastroenterol. 2011;46: 1081-1091.

5. Yoon JY, Park SJ, Hong SP, Kim TI, Kim WH, Cheon JH. Correlations of C-reactive protein levels and erythrocyte sedimentation rates with endoscopic activity indices in patients with ulcerative colitis. Dig Dis Sci. (Epub ahead of print). doi:10.1007/ s10620-013-2907-3.

6. Langhorst J, Elsenbruch S, Koelzer J, Rueffer A, Michalsen A, Dobos GJ. Noninvasive markers in the assessment of intestinal inflammation in inflammatory bowel diseases: performance of fecal lactoferrin, calprotectin, and PMN-elastase, CRP, and clinical indices. Am J Gastroenterol. 2008;103:162-169.

7. Schoepfer AM, Beglinger C, Straumann A, Trummler M, Renzulli P, Seibold F. Ulcerative colitis: correlation of the Rachmilewitz endoscopic activity index with fecal calprotectin, clinical activity, C-reactive protein, and blood leukocytes. Inflamm Bowel Dis. 2009;15:1851-1858.

8. Schoepfer AM, Beglinger C, Straumann A, et al. Fecal calprotectin more accurately reflects endoscopic activity of ulcerative colitis than the Lichtiger Index, C-reactive protein, platelets, hemoglobin, and blood leukocytes. Inflamm Bowel Dis. 2013;19:332-341.

9. Samuel S, Bruining DH, Loftus EV Jr, et al. Validation of the ulcerative colitis colonoscopic index of severity and its correlation with disease activity measures. Clin Gastroenterol Hepatol. 2013;11:49-54.

10. Travis SP, Schnell D, Krzeski P, et al. Reliability and initial validation of the ulcerative colitis endoscopic index of severity. Gastroenterology. 2013;145:987-995. 